\title{
Rapid Holographic Display of 3D Nanomaterials
}

Jacob Pietryga ${ }^{1}$, Jonathan Schwartz ${ }^{1}$, Talal Alothman ${ }^{2}$ and Robert Hovden ${ }^{1}$

${ }^{1}$ Department of Materials Science and Engineering, University of Michigan, United States, ${ }^{2}$ University of Michigan, United States

Electron tomography reveals a specimen's entire internal and external morphology [1,2], but the experimental acquisition and the subsequent volumetric reconstruction is a time-consuming process that often requires days to complete. Immediate assessment of 3D structure while operating the electron microscope is imperative for assessing and adjusting scientific objectives on-the-fly. Here we demonstrate rapid holographic display of 3D nanomaterials using electron microscopy. Seemingly physical 3D representations of specimens appear next to the microscope in a process that occurs under 3 minutes and with a single click. Holographic displays transform volumetric datasets into a 3D experience for all scientists in a room without reliance on 3D glasses or virtual reality headsets.

We demonstrate holographic display using helical nanoparticles made from a cadmium $(\mathrm{Cd})$ and a cysteine (Cys) organic framework. These nanoparticles' tunable helical structure dictates the macroscopic chiroptical activity in the near-infrared [3]. Knowing a particle's chirality is key to understanding the nanoparticles' light-emitting properties for optoelectronics. This holographic visualization immediately reveals the left-handedness of the $\mathrm{Cd} / \mathrm{Cy}$ nanoparticle and the $\sim 100 \mathrm{~nm} 3 \mathrm{D}$ spacing between petals at the tips of the nanoparticle - these features are not discernible using a single projection image.

Convincing holographic visualizations are made possible by leveraging holographic display hardware coming to market. Holographic displays are a novel technology that use an array of cylindrical lenses known as a "lenticular lens" that refract pixels' to distinct viewing directions (Fig. 1a) [4,5]. Projecting 45 unique images (Fig.1b) to each angle throughout the view frustum presents nanomaterials in a threedimensional space that feels natural to researchers (Fig. 1c,d) [6]. Currently, holographic visualizations have a limited-range $\left(\sim 44^{\circ}\right)$ light field with 572 x 322 rays. Thus, 3D structure can only be interpreted within this viewing range and display resolution [5,7].

Rapid holographic display of 3D nanomaterials consists of three main steps: data collection, data restructuring, and holographic presentation. Data collection is similar to tomographic experiments where projection images are collected along multiple angles. However, the range of tilts needed for rapid holographic display is only $+/-22^{\circ}$ at $1^{\circ}$ tilt increments, meaning data collection is substantially reduced from tomographic experiments (Fig. 2b). In the $\mathrm{Cd} / \mathrm{Cys}$ nanoparticle demonstration, 45 projection images (512 x 512 pixels) with a dwell time of $4 \mu \mathrm{s}$ were collected from $-22^{\circ}$ to $+22^{\circ}$ within 2 minutes at a total dose of $297.45 \mathrm{e} / \AA 2$. High acquisition speed is facilitated by setting the stage to eucentric height and automating stage tilt and image collection. The nanoparticles were examined using a FEI Talos operated at $200 \mathrm{keV}$ in STEM mode (convergence $=10.5 \mathrm{mrad}$ ) with an annular dark field detector (inner collection angle $=44 \mathrm{mrad}$ ) (Fig. 2a). Next, the set of projection images are restructured using a sub-pixel shader that maps each tilt angle to particular pixels on the display (Fig. 2d) [5]. The holographic display then reprojects the ADF-STEM images to defined angles (Fig. 2e). Because this reprojection occurs in hardware, the $3 \mathrm{D}$ visualization is immediate without requiring arduous tomographic reconstruction. Holographic display of 3D nanomaterials offers rapid understanding of specimens during a microscope session; however, interpretation of these life-like 3D representations is limited to a $44^{\circ}$ degree viewing 
range. In this sense, holographic display should be a compliment to electron tomography which offers complete quantification of a specimen's volumetric structure obtained after experimentation is complete.
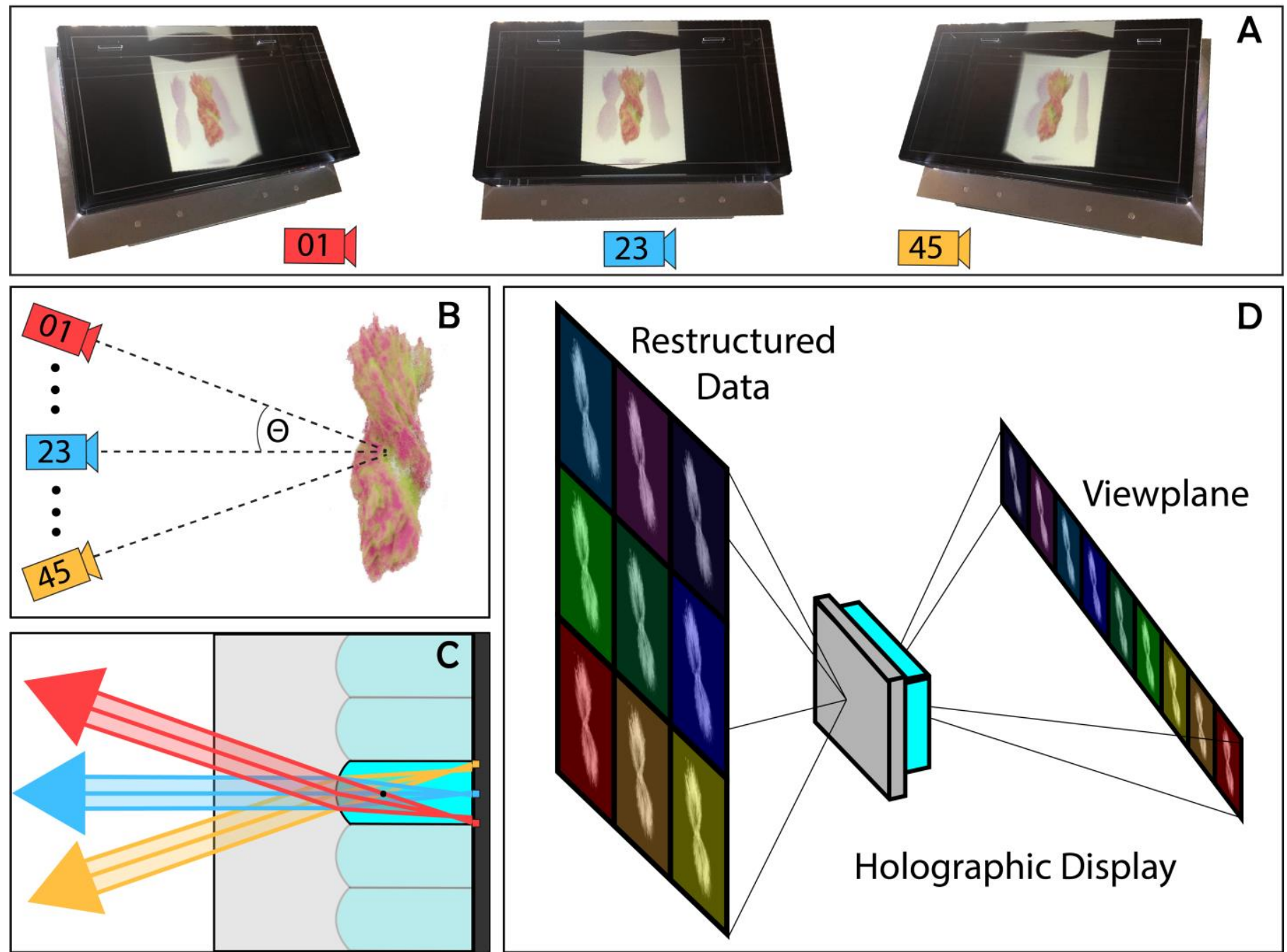

Figure 1. Figure 1 | STEM tomography of Cadmium-Cysteine nanoparticle visualized on holographic display. (A) Visual representation of the multiview display observed from extreme angles. Depth information is conveyed through lighting and shadows as well as relative position and size [8,9]. (B) Usage of holographic displays begins with generating 45 unique views focused on a point on the sample. The maximum viewing semi-angle $\Theta$ is $\sim 22^{\circ}$. (C) A single cylindrical lens's ray diagram shows how pixels project to viewing angles. (D) A holographic display projects distinct images into certain view regions along a viewplane. A viewer will perceive an image on the holographic display based on their position along the viewplane. 


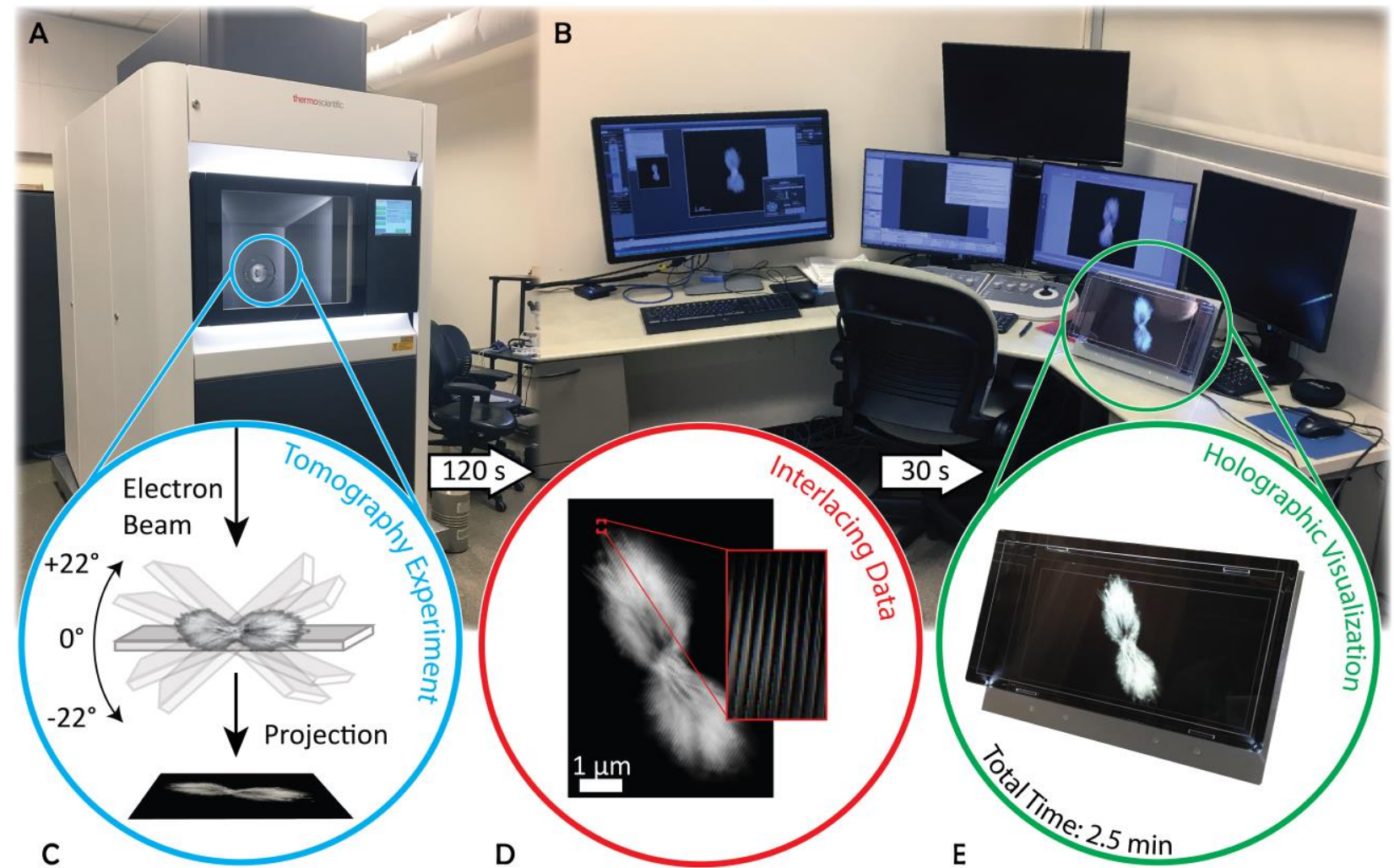

Figure 2. Figure 2 | STEM microscopy and rapid holographic inspection of Cadmium-Cysteine nanoparticle. (A) A script-controlled electron microscope automates the acquisition of projection images. (B) The holographic display fits natively along other displays in a microscope workstation. (C) A STEM collects 45 projection images across $+/-22^{\circ}$ specimen tilts. (D) All micrographs are interlaced together, assigning pixel placement based on angle for the holographic display. An example using the twisted specimen is shown with a close up of the interlace. (E) Final render of the bowtie displayed on LookingGlass holographic display. Binocular cues and intuitive perception of multiple views elucidate 3D specimen morphology.

\section{References}

[1] De Rosier, D., Klug, A. (1968). Reconstruction of Three Dimensional Structures from Electron Micrographs. Nature 217, 130-134.

[2] Hovden, R., \& Muller, D. (2020). Electron tomography for functional nanomaterials. MRS Bulletin, 45(4), 298-304.

[3] Yan, J., et al. (2020). Self-Assembly of Chiral Nanoparticles into Semiconductor Helices with Tunable near-Infrared Optical Activity. Chemistry of Materials. 32 (1), 476-488.

[4] Holliman, Nick. (2002). 3D Display Systems. 38.

[5] LookingGlass Factory (2020). Looking Glass 15.6” Development Kit \& 15.6” Pro.

[6] Frayne, S., Fok, Y.T., Lee, S.P. (2019). Superstereoscopic display with enhanced off-angle separation (U.S. Patent No. 10298921) U.S. Patent and Trademark Office. https://patents.justia.com/patent/10298921

[7] Woods, Andrew. (2012). Crosstalk in stereoscopic displays: a review. J. Electron. Imag. 21(4) 040902. 
[8] Lovell, P. G., Bloj, M., Harris, J. M.. (2012). Optimal integration of shading and binocular disparity for depth perception. Journal of Vision;12(1):1

[9] Goldstein, E.B. (1989). Sensation and Perception, 3rd ed. Belmont, CA: Wadsworth 